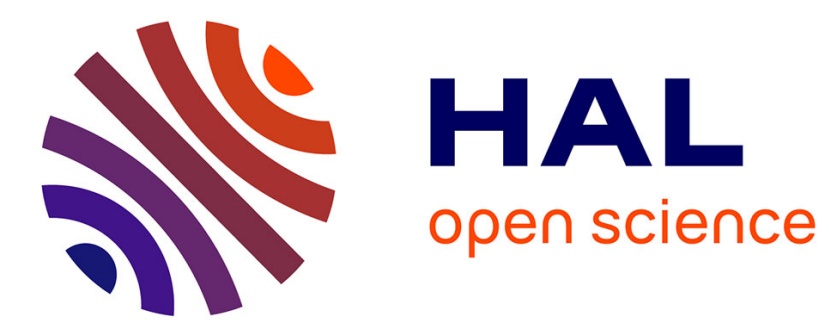

\title{
Smart pressure distribution estimation in biological joints for mechanical bio-inspired design
}

Elia Picault, Emmanuel Mermoz, Thomas Thouveny, Jean-Marc Linares

\section{To cite this version:}

Elia Picault, Emmanuel Mermoz, Thomas Thouveny, Jean-Marc Linares. Smart pressure distribution estimation in biological joints for mechanical bio-inspired design. CIRP Annals - Manufacturing Technology, 2018, 67 (1), pp.153 - 156. 10.1016/j.cirp.2018.04.116 . hal-01898672

\section{HAL Id: hal-01898672 \\ https://hal.science/hal-01898672}

Submitted on 18 Oct 2018

HAL is a multi-disciplinary open access archive for the deposit and dissemination of scientific research documents, whether they are published or not. The documents may come from teaching and research institutions in France or abroad, or from public or private research centers.
L'archive ouverte pluridisciplinaire HAL, est destinée au dépôt et à la diffusion de documents scientifiques de niveau recherche, publiés ou non, émanant des établissements d'enseignement et de recherche français ou étrangers, des laboratoires publics ou privés. 
Smart pressure distribution estimation in biological joints for mechanical bio-inspired design

\author{
Elia Picault ${ }^{\mathrm{a}}$,Emmanuel Mermoz (3) ${ }^{\mathrm{a}, \mathrm{b}}$, Thomas Thouveny ${ }^{\mathrm{a}}$, Jean-Marc Linares (1) , $^{\mathrm{a}, *}$ \\ a Aix Marseille Univ, CNRS, ISM, Marseille, France \\ b AIRBUS HELICOPTERS, Aéroport de Marseille Provence, Marignane 13700, France
}

Designing new mechanical links using bio-inspiration requires the knowledge of operating contact pressure in biological joints. However, the contact pressure magnitude and distribution are difficult to measure experimentally without disrupting the functioning of the articulation. In this paper, a new methodology to estimate the pressure distribution in biological joints is presented. A robust finite element model was developed based on in-vitro precise measurements of shapes, relative positions and loads in order to get accurate results. Furthermore, the envelope of the contact area was obtained through thermal imaging for comparison with the numerical results and qualitative validation of the FE model.

Biologically inspired design, Finite element method, Mechanical connection

\section{Introduction}

\subsection{Mechanical bio-inspired design}

Current mechanical connections technology based on generic links and simple geometries have reached their limits within the framework of severe loads despite the application of surface treatments to improve mechanical properties. Figure 1a shows a highly stressed pivot axis on which the contact area is severely damaged. A new path in research opened these last years, based on bio-inspiration and biomimicry [1,2]. Bio-inspired design could be a way to improve mechanical connections lifespan.

\subsection{Numerical modelling of biological joints}

To understand the functioning of biological joints, either for medical applications or in the context of mechanical bio-inspired design, numerical models are widely used. The finite element (FE) method was first applied to biological joints in the 80's with the study of the human knee [3-5]. Nowadays, the standard in medical biomechanics is anatomically based subject-specific 3D model. In those models, fluids and most soft tissues other than ligaments are almost always neglected. Secondary bones, ligaments, articular cartilage and disks are often neglected too. Bone is routinely assumed to be linear elastic isotropic and homogeneous although sometimes modelled as rigid or anisotropic. The same is true for articular cartilage which in practice is found to be nonlinear viscoelastic and heterogeneous. Some rare studies that focus on the role of articular cartilage consider part of this complex behaviour [5]. Finally, ligaments were often modelled as 1D nonlinear springs but tend to be represented by transversely isotropic hyper-elastic elements [6]. Geometries of bones and cartilage are usually acquired with computed tomography (CT) or magnetic resonance imaging (MRI), occasionally with digitizing systems [7]. Mechanical properties of bone are mostly derived from CT or MRI scans, whereas those of soft tissues are obtained from literature or by fitting the model to experimental data. In medical biomechanics, the main issues with FE models of biological joints are a poor knowledge of in-vivo loading and boundary conditions [4], as well as uncertainty of mechanical properties of biological materials (high variability among specimens and difficulties to obtain significant samples of fresh biological material) [5]. Biological geometry generation and meshing is also a sensitive aspect which requires a certain expertise due to the surfaces complexity, the low thickness of some components (e.g. cartilage) and the compromise between accuracy and computation time that must be achieved (especially for computer-assisted surgery). In the larger field of mechanical bio-inspired design, other issues arise due to the difficult access to biological material and to medical imaging equipment, as well as a lack of data on non-human biological links in the literature.

\subsection{Scope of the study}

In this context of mechanical bio-inspired design, our aim is to study the contact pressure magnitude and distribution in biological joints with no access to medical equipment. The present paper has a twofold purpose. First, it proposes a new methodology to develop a robust FE model of an articulation and presents a qualitative comparison between results obtained numerically and experimentally. Second, it provides a first investigation of the tested biological joint behaviour from a mechanical point of view. This methodology has been used to model a lamb elbow joint (Figure 1b). This choice results from a compromise between the type of link aimed for the bio-inspired design study (comparable to a pivot link) and the accessibility of biological material. The FE model accounts for humerus and radius-ulna bones (Figure 1c) with articular cartilage, whereas soft tissues have been neglected. In numerical study of contact, the results quality strongly depends on good representation of the bodies' shape and relative positions, as well as on the applied load. Therefore, an optical 3D scanner and a multi-component dynamometer are employed to obtain accurate geometrical and load data. Knowledge of in-vivo loading conditions is difficult to achieve however. Thus, this work is limited to a preliminary study of contact distribution, without seeking for the applied loads to be perfectly representative of those seen in-vivo. Doing so it is assumed that the contact area in an articulation is dictated first by the geometry and second by the load. Contact pressure magnitude and distribution are also difficult to measure experimentally without disrupting the joint behaviour. In literature, the main approach used to that end is the insertion of pressure sensitive films in the joint. However, this approach is intrusive, ill-suited for curved surfaces and gaps around 10 to $20 \%$ between real and measured contact area and magnitude can be obtained [8]. Here a thermal camera is used to estimate the contact area envelope based on local heating of the surface.
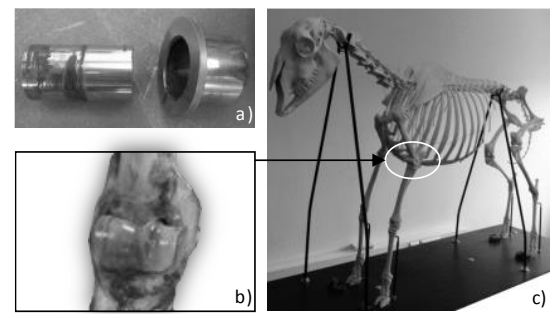

Figure 1. a) Damaged pivot axis. b) Sheep elbow joint with radius-ulna active surface inside humerus. c) Sheep skeleton with elbow joint. 
In the next section, the methodology is described in detail. First, the experimental set up and measurement process are presented. Then, the numerical process leading to the FE model is explained. Finally, the obtained results are compared and analysed.

\section{Principle of experimental setup and finite element model}

\subsection{Methodology overview}

\begin{tabular}{|c|c|}
\hline Experimental process & Numerical process \\
\hline Scan of experimental setup & Volumes construction \\
\hline Joint placement & Bodys placement \\
\hline Load a cquisition and & Load vectors computation \\
\hline Joint $30 \operatorname{scan}(\mathrm{statc})$ & Mesh and material definition \\
\hline acquisitions (dynamic) & Loads and $B C$ definition \\
\hline Scan of bones & Quasi static FE analysis \\
\hline
\end{tabular}

The methodology detailed hereafter is summarized in Figure 2. An experimental test bench is set up to run several tests (static and cyclic) on the articulation. Various measures are made before, during and after the tests to collect the data necessary for the FE model construction and validation. Next, a number of preliminary stages in the model construction are carried out, beginning with bones and cartilages reconstruction, their relative positioning and the computation of the load seen by the articulation in agreement with the previous measurements. Finally, the FE model is built with the meshing of the reconstructed geometries and the definition of materials, loads and boundary conditions.

\subsection{Experimental process}

The experimental setup is shown in Figure 3. The humerus of the biological joint (1) is sealed in a flange (2) mounted on a KISTLER dynamometric platform (3), itself fixed on a support (4). A system of pulleys and wires (5) links the radius-ulna (referred to as radius hereafter) to weights (6) on one side and to an operating handle on the other side (not visible on Fig. 3). The handle can be fixed at several positions on the wire to adjust the radius lead angle for static loading (Table 1) or it can be actuated by a tester to simulate passive flexion-extension at a standardized rate similar to that of gait. For both loading scenarios, the first step (before placing the joint to limit its drying) is to scan several markers placed on the dynamometric platform and its support using a GOM ATOS 3D optical scanner (7). Three markers on the lateral side (3a) of the platform, one on its frontal side (3b) and one on the support (4) will be used afterward to define the numerical model global reference frame. The articulation is then fixed on the platform and the wires are attached to the radius (without weights). Static tests are performed first. The handle is adjusted in position and the dynamometric platform is started in order to record the load seen by the joint with a resolution of $0.05 \mathrm{~N}$. A first part of the acquisition $(35 \mathrm{~s}$ at $300 \mathrm{~Hz})$ is realized unloaded to detect the drift of the platform. Then an $8 \mathrm{~kg}$ weight (approximately one quarter of a lamb weight) is set up and acquisition continues during a time sufficient for the system to stabilize $(120$ to $180 \mathrm{~s}$ at $300 \mathrm{~Hz})$. Simultaneously, several components of the test bench are scanned to obtain the radius position and the load directions relative to the global reference frame. To that end, additional elements allowing an optimal placement of markers detected by the optical scanner are introduced on the wires (8) and the radius (9). The same process is repeated for each inclination of the radius. For cyclic testing, the operating handle is actuated to operate around one hundred cycles ( 5 cycles per 10 s during 240s) and a FLIR thermal camera (10) is used to record the temperature on the humerus surface with a sensitivity of $0.05^{\circ} \mathrm{C}$. To compare afterward the 2D thermal image and the 3D FE model with the proper orientation, the thermal camera is partially digitalized. However, to know its position in the global reference frame it is necessary to scan also a physical path between the camera and the test bench. Though a metal straight (11) with markers is added to the experimental setup. After the tests, the articulation is dismantled and active surfaces are digitalized. The humerus is scanned sealed in the flange (2) itself fixed on the dynamometric platform (3), whereas the radius-ulna is scanned with the plastic cylinder (9) attached.

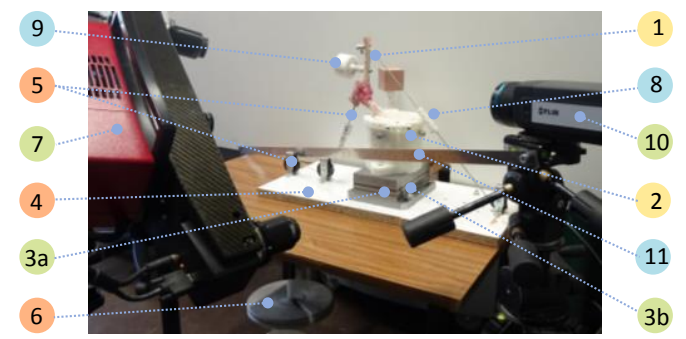

Figure 3. Experimental setup. 1) Biological joint. 2) Flange. 3a) Lateral side and 3b) frontal side of dynamometric platform. 4) Support. 5) Wires and pulleys system. 6) Weight. 7) 3D optical scanner. 8) Plastic cylinder with markers (load direction). 9) Plastic cylinder with markers (radius local reference frame). 10) Thermal camera. 11) Metal straight with markers (physical path between test bench and thermal camera).

\subsection{Numerical process}

Using the data measured from the experimental set up, the finite element model is then built in several steps. First, CATIA V5 is used to generate part files containing the global and local reference frames, the humerus and radius bones and cartilages plus the load axes. The bone and cartilage parts (Figure 5a) are created using the following procedure. The average external surfaces generated by the optical scanner software for the bones, which are 3D open tessellated surfaces, are converted to closed BSpline surfaces and smoothed before being used to create volume parts. The articular cartilage is created using the Thick Surface tool. The cartilaginous surfaces are manually bonded on the parts based on pictures of the fresh bones. A thickness of $0.15 \mathrm{~mm}$ based on literature values is then defined in the internal (bone) direction and a Boolean operation ensures the conformity of each bone/cartilage couple. For the load directions, the plastic cylinders fixed on the wires are reconstructed in CATIA and their axes of revolution are determined using the Feature Recognition tool. Finally, a product file is generated for each static test with the bones, cartilages and load directions placed according to the experimental setup using the global and local frames. Each product file is then imported in NX 11 to build the associated FE model. Two isotropic elastic materials are defined for bone (cortical and trabecular bones are not differentiated) and cartilage. For both material, mechanical properties are obtained from literature. The values of Young's modulus and Poisson's ratio respectively are $18 \mathrm{GPa}$ and 0.3 for bone, and $10 \mathrm{MPa}$ and 0.45 for articular cartilage. 


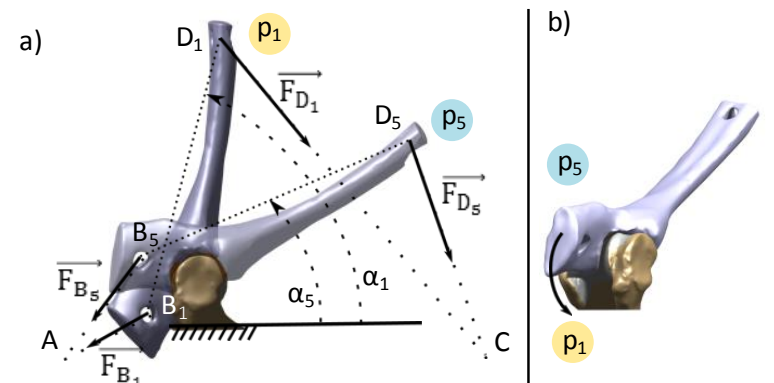

Figure 4. a) Extremal inclinations for static load cases (points $A$ and $C$ represent the wire-pulley contact points). b) Back view of the articulation.

Table 1. Prescribe radius-ulna inclination and load values for static tests (Figure 4 for definition of used notations)

\begin{tabular}{|c|c|c|c|}
\hline Angular position & $\left\|\mathrm{F}_{\mathrm{Bi}}+\mathrm{F}_{\mathrm{Di}}\right\|_{\exp }$ & $\mathrm{F}_{\mathrm{i}}=\left\|\mathrm{F}_{\mathrm{Bi}}+\mathrm{F}_{\mathrm{Di}}\right\|_{\text {num }}$ & Deviation \\
\hline$\alpha_{1}=73^{\circ}$ & $60.73 \mathrm{~N}$ & $62.51 \mathrm{~N}$ & $2.92 \%$ \\
\hline$\alpha_{2}=61^{\circ}$ & $68.88 \mathrm{~N}$ & $70.81 \mathrm{~N}$ & $2.81 \%$ \\
\hline$\alpha_{3}=49^{\circ}$ & $65.49 \mathrm{~N}$ & $66.58 \mathrm{~N}$ & $1.66 \%$ \\
\hline$\alpha_{4}=38^{\circ}$ & $66.17 \mathrm{~N}$ & $67.02 \mathrm{~N}$ & $1.28 \%$ \\
\hline$\alpha_{5}=27^{\circ}$ & $67.36 \mathrm{~N}$ & $67.44 \mathrm{~N}$ & $0.11 \%$ \\
\hline
\end{tabular}

Bones are meshed with 10,7360 linear tetrahedral elements to limit computation cost, and cartilage with 39,254 quadratic hexahedral elements more suited for contact problems (Figure $5 \mathrm{~b})$. Bone and cartilage meshes are coupled using rigid 1D elements. Frictionless contact is defined between both articular cartilages external surfaces (humerus cartilage being the master surface). The humerus is embedded, whereas the radius is subjected to two follower forces applied at points B and D (Figure $4 a)$. For each static test, load values (Table 1 ) are computed using rigid body statics knowing the loads direction and their reduction at the dynamometric platform origin and corrected to obtain the static balance of the FE model. SAMCEF MECANO is used to run the quasi-static FE analysis (2.5h computation time per test).

\section{Results}

The experimental results were used for a qualitative validation of the numerical model, which was itself used for a first investigation of the articulation behaviour. The contact area distribution (with its impact on stability) and the maximum contact pressure were compared for each static test. The position with the most interesting contact parameters was determined.

\subsection{FE model qualitative validation}

During cyclic test, the thermal camera films the heating of the humerus active surface (the radius surface is not visible). At the end of the test, the temperature has risen on three parallel strips and oriented in the movement direction (Figure 5c). It should be noted that the main source of heating is not friction (cartilage/cartilage friction coefficient is around 0.01), but most probably the viscoelastic hysteresis behaviour of the cartilage.
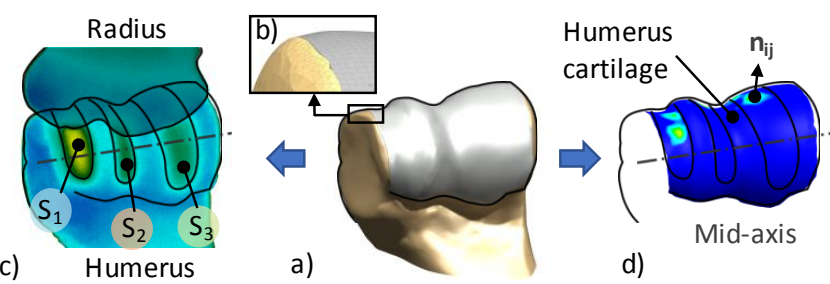

Figure 5. a) Humerus head (beige: bone - grey: cartilage) reconstructed in Catia V5 from 3D optical scans. b) Mesh generated in NX 11. c) Thermal image of humerus and radius at the end of the cyclic test. d) Contact pressure on humerus cartilage obtained with the FE model for position $\alpha_{4}$.
For each of the five static positions, the FE model predicts between six to seven contact areas on the humerus active surface (Figure 6). The contact areas location is relatively stable from a position to the other on the humerus as it is in fixed position regarding the experimental loads. The larger zones with higher contact pressures are located on top of the humerus head $\left(\mathrm{Z}_{1}\right.$ to $Z_{5}$ on Fig. 6) and fit into the three stripes seen on the thermal image and extrapolated to the area masked by the radius (Figure $5 \mathrm{~d})$. Another one, which is smaller with a low contact pressure and might result from a defect in the numerical geometry, can be seen behind the humerus head $\left(\mathrm{Z}_{6}\right.$ on Fig. 6). The last one $\left(\mathrm{Z}_{7}\right)$ appears only for positions 1 (hyperextension) to 4 (partial flexion) when the protrusion of the radius-ulna comes to fit into the hollow of the humerus as shown in Figure 4b. This contact area makes an additional axial stop which secure the joint when the leg is tightened, for example during the stance phase of gait.

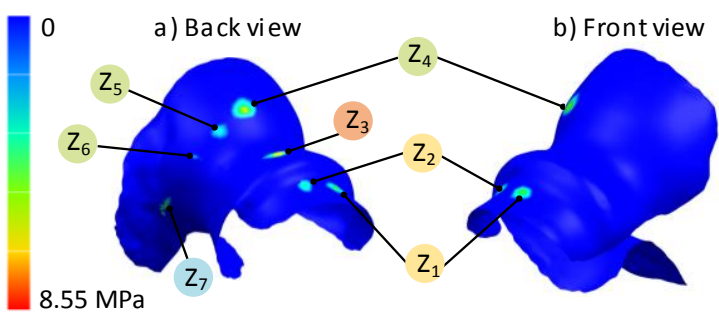

Figure 6. Contact pressure on humerus head obtained with the FE model for position $\alpha_{4}$. View from behind a) and from front $\mathbf{b}$ ).

At the sight of the thermal image, it could have been expected to obtain fewer and larger contact zones. Nevertheless, these results are not contradictory. Indeed, whereas the contact zones on the humerus are relatively stable, those on the opposite surface evolves as the radius orientation changes. The superposition of the contact zones on the radius cartilage thus corresponds to three relatively parallel strips as on the thermal image. The radius surface warmed up along these strips due to the cartilage compression, warms back the opposite humerus surface on an area which is thus wider than the real contact area.

\subsection{Biological joint study}

\subsubsection{Validation of the kinematic model}

The geometry and functioning of this biological joint let think that it could be assimilated to a pivot link. To confirm this hypothesis, two points had to be validated. In one hand, the presence of axial stops which are ensured here by the contact zones $\mathrm{Z}_{3}$ to $\mathrm{Z}_{6}$ whatever the position $\mathrm{p}_{i}$ is, and strengthened by the zone $\mathrm{Z}_{7}$ for the most open positions. And in the other hand, the fact that the cartilaginous surface providing the rotational guidance can be assimilated to a surface of revolution. For this second point, the area of the humerus cartilage in which is situated the contact zone $\mathrm{Z}_{7}$ (which serves as axial stop only) was considered as not being a part of the guidance surface. A mid-axis (Figure $5 \mathrm{~d}$ ) in the sense of the least squares was then determine to this delimited surface with a maximal error bar of $0.73 \mathrm{~mm}$ (Figure 7a). This value being low, this surface can be compared to a surface of revolution.

\subsubsection{Contact zones distribution}

After validating the kinematic model, the distribution of the contact zones was investigated. First, the radius associated with every node of the mesh situated in one of the contact zones $\mathrm{Z}_{\mathrm{j}}$ (excluded $\mathrm{Z}_{7}$ ) was computed for each position $\mathrm{p}_{\mathrm{i}}$. Figure $7 \mathrm{~b}$ represents the mean value obtained for all the contact zones as well as the upper and lower bounds with 95\% confidence. The mean radius for all positions is equal to $10.23 \mathrm{~mm}$. 

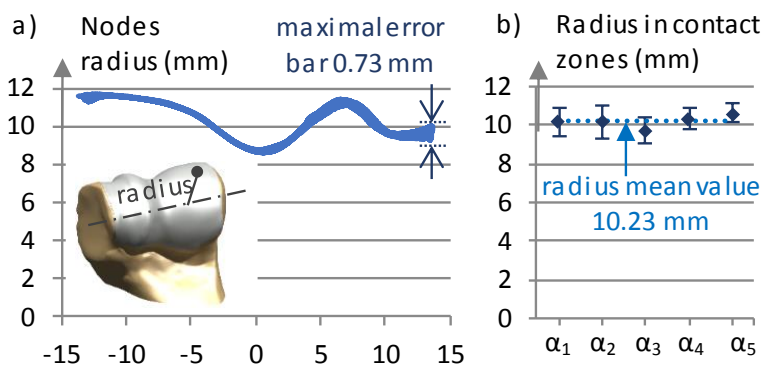

Figure 7. a) Radius of all nodes in the active surface plotted in the same plane with maximal error bar. b) Contact radius mean value and error bars for $95 \%$ confidence for each position $\alpha_{\mathrm{i}}$.

Table 2. Joint stability indicator $\operatorname{det}\left(\mathrm{A}_{\mathrm{i}}\right)$, maximal contact pressure value (as it is and normalized) and localisation for the five positions $\alpha_{\mathrm{i}}$.

\begin{tabular}{|c|c|c|c|c|c|}
\hline & $\alpha_{1}$ & $\alpha_{2}$ & $\alpha_{3}$ & $\alpha_{4}$ & $\alpha_{5}$ \\
\hline $\operatorname{det}\left(\mathbf{A}_{\mathrm{i}}\right)$ & -3.80 & -19.34 & -37.87 & -39.69 & -20.37 \\
\hline $\mathrm{P}_{\mathrm{i}, \max }(\mathrm{MPa})$ & 22.37 & 8.95 & 8.55 & 8.18 & 11.66 \\
\hline $\mathrm{P}_{\mathrm{i}, \max } \cdot \mathrm{F}_{\operatorname{moy}} / \mathrm{F}_{\mathrm{i}}(\mathrm{MPa})$ & 23.93 & 8.45 & 8.59 & 8.16 & 11.56 \\
\hline Zone & $\mathrm{Z}_{3}$ & $\mathrm{Z}_{3}$ & $\mathrm{Z}_{3}$ & $\mathrm{Z}_{3}$ & $\mathrm{Z}_{3}$ \\
\hline
\end{tabular}

Then, the joint stability was estimated for each position $\alpha_{i}$ by calculating a quantity $\operatorname{det}\left(\mathbf{A}_{\mathbf{i}}\right)$. Equation 1 shows the matrix $\mathbf{A}_{\mathbf{i}}$. This calculation was made by taking into account only the five main contact areas, the sixth contact zone being negligible compared to the five others. A local frame $(0, \mathbf{x}, \mathbf{y}, \mathbf{z})$ was defined, whose vector $\mathbf{x}$ corresponds to the mid-axis computed beforehand. For each of the five contact zones $\mathrm{Z}_{\mathrm{j}}$, the position of the nodes in the global frame of the FE model are collected and transported in the local frame. Also, the barycentre $\mathrm{M}_{\mathrm{ij}}$, a midplane in the sense of the least squares containing the barycentre and the normal $\mathbf{n}_{\mathrm{ij}}$ to this plane were computed for each of these zones (Figure 5d).

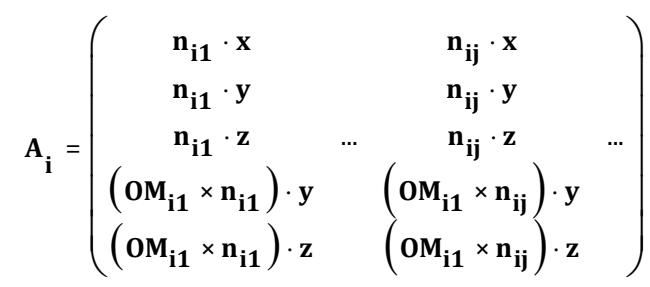

The columns of matrix $\mathbf{A}_{\mathbf{i}}$ correspond to the Plücker's coordinates of the barycenters $\mathrm{M}_{\mathrm{ij}}$ and its lines to the fixed degrees of freedom of the joint (all excepted the rotation around the mid-axis $\mathbf{x}$ ).

In the case of a connection constituted of rigid bodies for which it is possible to apply the fundamental principle of dynamics, the following relation can be written: $\mathbf{A}_{\mathbf{i}} \cdot \mathbf{R}_{\mathbf{i}}=\mathbf{0}$ with $\mathbf{R}_{\mathbf{i}}=\left\{\mathrm{R}_{\mathrm{i} 1} \ldots \mathrm{R}_{\mathrm{ij}} \ldots\right\}^{\mathrm{T}}$ where the notation $\mathrm{R}_{\mathrm{ij}}$ corresponds to the norm of the reaction vector (directed along the normal $\mathbf{n}_{\mathbf{i j}}$ ) in the contact zone $\mathrm{Z}_{\mathrm{j}}$ for the position $\alpha_{i}$. If $\operatorname{det}\left(\mathbf{A}_{\mathbf{i}}\right)=0$ the system is hyperstatic, otherwise it is isostatic. In this second case, the greater $\left|\operatorname{det}\left(\mathbf{A}_{\mathbf{i}}\right)\right| \mid \operatorname{det}\left(\mathbf{A}_{\mathrm{i}}\right) \|$ is, the more stable the system is. Although the bones and cartilage are deformable bodies, the obtained value can discriminate the most stable position among the five that have been tested (the contact geometry not being identical for all the positions since the surfaces of both bones are not perfect surfaces of revolution). The results are presented in Table 2 and show that the most stable position is the 4 th.

\subsubsection{Study of the maximum contact pressure}

Finally, the maximal value and location of the contact pressure were compared for each position (Table 2). It can be noticed that the maximal pressure (normalized according to the norm of the applied load) evolves almost reversely to the stability (greater is the stability, lower is the pressure). The lower maximal pressure is reached in position $\alpha_{4}$ which is also the most stable.

\section{Conclusion and outlook}

This study of contact within a biological joint in a bio-inspired design perspective was a first stage that led us to propose a methodology to build a FE model of the articulation fed by experimental data obtained with relatively accessible facilities for mechanics. This methodology ensures the good positioning of the articulation and the adequacy of the load with regard to the experimental test, and validate qualitatively the location of the contact zones obtained with the FE model. A first investigation of the biological joint behaviour from a mechanical point of view was made thanks to this model. It seems that the Nature, despite the complex geometry of the articular surfaces, placed the contact zones on the same radius which minimizes the kinematic heterogeneousness and thus the loss of energy during movement. Furthermore, the distribution of the contact zones as well as the orientation of their normal seem to correspond to an isostatic system. Thus, it should be possible to draw inspiration from it in the design of a mechanical connection of standard precision, which would not be the case of an hyperstatic system. To confirm these results, future work will aim at studying the evolution of the contact zones depending on the load.

\section{Acknowledgements}

This work was supported by Airbus Helicopters and AixMarseille University. The experimental devices were founded by: European Community, French Ministry of Research and Education and Aix-Marseille Conurbation Community. Research versions of NX 11 and CATIA V5 were used in this work.

\section{References}

[1] Shu, L. H., Ueda, K., Chiu, I., Cheong, H., 2011, Biologically inspired design, Annals of the CIRP, 60/2:673-693.

[2] Malshe, A., Rajurkar, K., Samant, A., Norgaard Hansen, H., Bapat, S., Jiang, W., 2013, Bio-inspired functional surfaces for advances applications, Annals of the CIRP, 62/2:607-628.

[3] Huiskes, R., Chao, E., 1983, A survey of finite element analysis in orthopedic biomechanics: The first decade, Journal of Biomechanics, 16/6:385-409.

[4] Taylor, M., Prendergast, P. J., 2015, Four decades of finite element analysis of orthopaedic devices: Where are we now and what are the opportunities? Journal of Biomechanics, 48:767-778.

[5] Marchi, B. C., Arruda, E. M., 2017, A study on the role of articular cartilage soft tissue constitutive form in models of whole knee biomechanics, Biomechanics and Modeling in Mechanobiology, 16:117-138.

[6] Weiss, J. A., Gardiner, J. C., Ellis, B. J., Lujan, T. J., Phatak, N. S., 2005, Threedimensional finite element modeling of ligaments: Technical aspects, Medical Engineering \& Physics, 27:845-861.

[7] Haut Donahue, T. L., Hull, M. L., Rashid, M. M., Jacobs, C. R., 2002, A finite element model of the human knee joint for the study of tibio-femoral contact, Journal of Biomechanical Engineering, 124:273-280.

[8] Liau, J-J., Cheng, C-K., Huang, C-H., Lo, W-H., 2002, Effect of Fuji pressure sensitive film on actual contact characteristics of artificial tibiofemoral joint, Clinical Biomechanics, 17:698-704. 